\title{
Unitary Evolutions and Horizontal Lifts in Quantum Stochastic Calculus
}

\section{David Applebaum}

Department of Mathematics, Statistics and Operations Research, Nottingham Polytechnic, Burton Street, Nottingham, NG1 4BU, England

Received July 30, 1989; in revised form January 18, 1991

\begin{abstract}
Unitarity is proved for a class of solutions of quantum stochastic differential equations with unbounded coefficients. The resulting processes are then used to construct algebraic quantum diffusions. Applications include an existence proof for a class of diffusions on the non-commutative two-torus and a geometric interpretation for diffusions driven by the classical Poisson process.
\end{abstract}

\section{Introduction}

Let $h_{0}$ be a complex, separable Hilbert space and $\Gamma$ be boson Fock space over $L^{2}([0, \infty))$. A major role in the development of quantum stochastic calculus has been played by processes $U=(U(t), t \geqq 0)$ which arise as the solutions of the following linear stochastic differential equation on $h_{0} \otimes \Gamma$ :

$$
\begin{aligned}
d U & =U\left(L_{1} \otimes d A^{\dagger}+L_{2} \otimes d \Lambda+L_{3} \otimes d A+L_{4} \otimes I d t\right), \\
U(0) & =I,
\end{aligned}
$$

where $L_{j} \in B\left(h_{0}\right)(1 \leqq j \leqq 4)$ and $A^{\dagger}, \Lambda$ and $A$ are the processes of creation, conservation and annihilation in $\Gamma$ (respectively) $([13,18])$. We note that the coefficients $L_{j}(1 \leqq j \leqq 4)$ do not here depend on time.

Of particular importance are the sub-class of solutions to (1.1) where the algebraic relations between the $L_{j}$ 's are such that $U$ is unitary operator valued. These unitary processes have found many applications including the construction of Markov dilations of quantum dynamical semigroups $([13,14])$, the description of quantum processes with stationary and independent increments over graded *-bialgebras [1] and the representation of time-ordered stochastic product integrals [11].

To the extent that these unitary processes may be regarded as solutions of a "Schrödinger equation in the presence of noise" [18], it is clear that the case where the $L_{j}$ 's are no longer bounded will be of great practical interest. So far, however, although existence of solutions to (1.1) was established in [13] there has been no 
rigorous analysis of the conditions under which these solutions are unitary except in the special case where the $L_{j}$ 's are linear combinations of the raising and lowering operators for the harmonic oscillator ([8]). The main purpose of the present paper is to provide such an analysis.

The motivation for this work has been the attempts by M. E. Evans and R. L. Hudson (e.g. [12,10]) and the present author [4] to obtain algebraic generalisations of the notion of a diffusion process on a smooth manifold. If such a manifold $M$ possesses an affine connection, the required diffusion may be obtained by the method of horizontal lifts which involves constructing an appropriate process on the tangent bundle over $M$ and extracting the diffusion from the induced motion on $M$ [16]. Motivated by the non-commutative differential geometry of A. Connes [6], an algebraic generalisation of the above procedure was outlined in [2] wherein it was argued that the tangent bundle should be replaced by an appropriate projective module $\mathscr{D}$ over the algebra of interest. In many cases $\mathscr{D}$ may be realised as a dense linear manifold in a Hilbert space on which the connections will act as unbounded linear operators which preserve $\mathscr{D}$. Our horizontal lifts are then driven by a "quantum stochastic parallel transport process" of the form (1.1), where the $L_{j}$ 's are not all bounded but still satisfy the algebraic conditions which guarantee unitarity in the bounded case.

Having demonstrated unitarity of our parallel transport process, we obtain as a corollary an existence proof for a large class of algebraic diffusions with unbounded coefficients. (The only previous result in this direction required boundedness [9].) In particular we obtain the first rigorous construction of the $(A, B)$-diffusions which were introduced in [4] to overcome the cohomological obstructions that limited the scope of the theory described in [12].

A field theoretic interpretation of quantum stochastic parallel transport is given in [3] and an application of quantum diffusions on the non-commutative two-torus to solid state physics is described in [19].

It should be noted that the definition of quantum stochastic parallel transport given below (Eq. 5.19) only agrees with the definition [2] when the " $d \Lambda$ term" is absent. With hindsight it is clear that the author was suffering in [2] from the disease of "Gaussian prejudice" of which he hopes he may now declare himself cured!

Notation. If $\mathscr{D}$ and $\mathscr{E}$ are dense linear manifolds in Hilbert spaces $h_{1}$ and $h_{2}$ (respectively) we denote their algebraic tensor product by $\mathscr{D} \otimes \mathscr{E}$.

For $T$ a linear operator on $h_{1}$ we denote its domain by $\operatorname{Dom}(T)$ and its range by $\operatorname{Ran}(T)$. When $T$ is closeable, we denote its closure by $\bar{T}$. The ampliation of $T$ in $h_{1} \otimes h_{2}$ is the operator $T \otimes I$ with domain $\operatorname{Dom}(T) \otimes h_{2}$. Note that if $T$ is closeable then so is $T \otimes I$ with $\overline{T \otimes I} \supseteqq \bar{T} \otimes I$. When there is no room for ambiguity, we will abuse notation and identify $T$ with its ampliation. $L(\mathscr{D}, \mathscr{E})$ will denote the linear space of closeable linear operators from $h_{1}$ into $h_{2}$ for which $T \in L(\mathscr{D}, \mathscr{E}) \Rightarrow$ $\operatorname{Dom}(T)=\mathscr{D}$ and $\operatorname{Ran}(T) \subseteq \mathscr{E}$. We will use $L(\mathscr{D})$ for the case where $h_{2}=h_{1}$ and $\mathscr{E}=\mathscr{D}$.

Consider the subspace $O(\mathscr{D})$ of $L(\mathscr{D})$ comprising those $T \in L(\mathscr{D})$ for which $\mathscr{D} \subseteq \operatorname{Dom}\left(T^{*}\right)$ and $T^{*} \mathscr{D} \subseteq \mathscr{D}$.

$O(\mathscr{D})$ is a ${ }^{*}$-algebra under the involution $T \rightarrow T^{\dagger}$, where $T^{\dagger}$ is the restriction of $T^{*}$ to $\mathscr{D}$. In fact, $O(\mathscr{D})$ is the maximal $O p^{*}$-algebra on $\mathscr{D}$ in the sense of [17]. 
Let $A$ be a *algebra and $d \in \mathbb{N}$. $A^{d}$ (respectively $M_{d}(A)$ ) will denote the linear space $A \otimes \mathbb{C}^{d}$ (respectively the ${ }^{*}$-algebra $A \otimes M_{d}(\mathbb{C})$ ). Note that $A^{d}$ (respectively $\left.M_{d}(A)\right)$ is a two-sided $A$-module where the module multiplication is pointwise on components (respectively matrix entries).

If $X$ is a vector or matrix then $X^{T}$ denotes its transpose. Now let $A$ and $B$ be *-algebras and $F$ a linear space such that $F$ is both a right $A$-module and a left $B$-module. Let $\sigma$ be a ${ }^{*}$-homomorphism from $A$ into $B$.

A $\sigma$-derivation is a linear map $\delta: A \rightarrow F$ satisfying

$$
\delta(a b)=\sigma(a) \delta(b)+\delta(a) b
$$

for all $a, b \in A$.

$\operatorname{Der}_{\sigma}(A, F)$ will denote the linear space of all such $\sigma$-derivations.

$Z(A)$ will denote the centre of the *-algebra $A$.

If $C \subseteq O(\mathscr{D})$ then

$$
C^{\dagger}=\left\{X \in O(\mathscr{D}) ; X=T^{\dagger} \text { for some } T \in C\right\} .
$$

\section{Preliminaries-Quantum Stochastic Integrals}

The main purpose of this section is to establish our notation and to state the main results of quantum stochastic calculus which we will use in the sequel. Proofs of these, in a form close to that given below may be found in [8] (see also the seminal paper [13]).

Fix $d \in \mathbb{N}$ and let $H$ denote the complex separable Hilbert space $L^{2}\left(\mathbb{R}^{+}, \mathbb{C}^{d}\right)$ which is naturally isomorphic to $L^{2}\left(\mathbb{R}^{+}\right) \otimes \mathbb{C}^{d} . \Gamma(H)$ will denote boson Fock space over $H$.

For $f \in H$, let $\psi(f)$ denote the corresponding exponential vector (coherent state) in $\Gamma(H)$. Note that for all $f, g \in H$, we have

$$
\langle\psi(f), \psi(g)\rangle=e^{\langle f, g\rangle} .
$$

Let $S$ be a dense linear manifold in $H$, then the set $E(S)$ which is the linear span of $\{\psi(f), f \in S\}$ is dense in $\Gamma(H)$. We will here take $S$ to consist of locally bounded functions.

We denote by $\left\{e_{j}, 1 \leqq j \leqq d\right\}$ the natural basis for $\mathbb{C}^{d}$ so that $e_{j}$ is the vector with 1 in the $j^{\text {th }}$ entry and zeroes elsewhere. The dual basis will be denoted by $\left\{e_{j}^{*}, 1 \leqq j \leqq d\right\} .\left\{I_{i j}, 1 \leqq i, j \leqq d\right\}$ will be the natural basis in $M_{d}(\mathbb{C})$ and its dual basis will be denoted by $\left\{I_{i j}^{*} ; 1 \leqq i, j \leqq d\right\}$.

For $t \in \mathbb{R}^{+}, 1 \leqq i, j \leqq d$ and $f \in S$, we define

$$
\begin{aligned}
& a_{j}^{\dagger}(t) \psi(f)=\left.\frac{d}{d \varepsilon} \psi\left(f+\varepsilon\left(\chi_{[0, t)} \otimes e_{j}\right)\right)\right|_{\varepsilon=0}, \\
& \Lambda_{i j}(t) \psi(f)=\left.\frac{d}{d \varepsilon} \psi\left(e^{\left(\varepsilon \chi_{[0, t)} \otimes I_{i j}\right)} f\right)\right|_{\varepsilon=0}, \\
& a_{j}(t) \psi(f)=\left\langle f, \chi_{[0, t)} \otimes e_{j}\right\rangle \psi(f) .
\end{aligned}
$$

Note that the pairs of operators $\Lambda_{i j}(t)$ and $\Lambda_{j i}(t)$ and (as the notation suggests), $a_{j}(t)$ and $a_{j}^{\dagger}(t)$ are mutually adjoint on the domain $E(S)$. 
Now let

$$
\begin{aligned}
A^{\dagger}(t) & =\sum_{j=1}^{d} a_{j}^{\dagger}(t) \otimes e_{j}^{*}, \\
\Lambda(t) & =\sum_{i, j=1}^{d} \Lambda_{i j}(t) \otimes I_{i j}^{*}, \\
A(t) & =\sum_{j=1}^{d} a_{j}(t) \otimes e_{j},
\end{aligned}
$$

$A^{\dagger}=\left(A^{\dagger}(t), t \geqq 0\right), \Lambda=(\Lambda(t), t \geqq 0)$ and $A=(A(t), t \geqq 0)$ are the processes of creation, conservation and annihilation (respectively) in $\Gamma(\bar{H})$.

Now let $h_{0}$ be a complex separable Hilbert space and $\mathscr{D}$ be a dense linear manifold in $h_{0}$. We write $h=h_{0} \otimes \Gamma(H)$ and for $1 \leqq i, j \leqq d, t \geqq 0$ let $E_{j}(t), F_{i j}(t), G_{j}(t)$ and $H(t)$ be linear operators on $h$, each with domain $\mathscr{D} \otimes E(S)$ such that the processes $\left(E_{j}(t), t \geqq 0\right),\left(F_{i j}(t), t \geqq 0\right),\left(G_{j}(t), t \geqq 0\right)$ and $(H(t), t \geqq 0)$ are adapted and square-integrable in the sense of [13].

For each $t \geqq 0$, define

$$
\begin{aligned}
& E(t)=\sum_{j=1}^{d} E_{j}(t) \otimes e_{j}, \\
& F(t)=\sum_{i, j=1}^{d} F_{i j}(t) \otimes I_{i j}, \\
& G(t)=\sum_{j=1}^{d} G_{j}(t) \otimes e_{j}^{*} .
\end{aligned}
$$

Let $E=(E(t), t \geqq 0), F=(F(t), t \geqq 0)$ and $G=(G(t), t \geqq 0)$.

Following [8] and [13] we may define stochastic integrals with respect to the ampliations of the integrators onto the whole of $h$ to be processes $M=(M(t), t \geqq 0)$ wherein each $M(t)$ is a linear operator on $h$ with domain $\mathscr{D} \otimes E(S)$ given by

$$
M(t)=M(0)+\int_{0}^{t}\left(d A^{\dagger} E+d \Lambda F+G d A+H d t\right)
$$

which may be written in component form

$$
M(t)=M(0)+\int_{0}^{t}\left(\sum_{j=1}^{d} E_{j} d A_{j}^{\dagger}+\sum_{i, j=1}^{d} F_{i j} d \Lambda_{i j}+\sum_{j=1}^{d} G_{j} d A_{j}+H d t\right) .
$$

$M$ is itself adapted and square-integrable. by

For $f, g \in S$, define the adapted square-integrable process $K_{f, g}=\left(K_{f, g}(t), t \geqq 0\right)$

$$
K_{f, g}(t)=\overline{f(t)}^{T} E(t)+\overline{f(t)}^{T} F(t) g(t)+G(t) g(t)+H(t) .
$$

For $u, v \in \mathscr{D}, t \geqq 0$ we have the following basic formula:

$$
\langle u \otimes \psi(f),(M(t)-M(0))(v \otimes \psi(g))\rangle=\int_{0}^{t}\left\langle u \otimes \psi(f), K_{f, g}(s)(v \otimes \psi(g))>d s .\right.
$$


For $X_{j}(1 \leqq j \leqq n)$ operators on $h$ with domain $\mathscr{D} \unrhd E(S)$ let

For $\psi \in \mathscr{D} \underline{\otimes}(S)$, define $X \psi \in h^{d}$ by

$$
X=\sum_{j=1}^{n} X_{j} \otimes e_{j}
$$

$$
X \psi=\sum_{j=1}^{n} X_{j} \psi \otimes e_{j}
$$

Now let $M_{1}$ and $M_{2}$ be stochastic integrals with

$$
d M_{p}=d A^{\dagger} E_{p}+d \Lambda F_{p}+G_{p} d A+H_{p} \quad(p=1,2) .
$$

Our second fundamental result is the quantum Itô formula:

$$
\begin{aligned}
\left\langle M_{1}(t)(u \otimes \psi(f)), M_{2}(t)(v \otimes \psi(g))\right\rangle= & \left\langle M_{1}(0)(u \otimes \psi(f)), M_{2}(0)(v \otimes \psi(g))\right\rangle \\
+ & \int_{0}^{t}\left\{\left\langle M_{1}(s)(u \otimes \psi(f)), K_{f, g}^{2}(s)(v \otimes \psi(g))\right\rangle\right. \\
+ & \left\langle K_{g, f}^{1}(s)(u \otimes \psi(f)), M_{2}(s)(v \otimes \psi(g))\right\rangle \\
+ & \left(\left[E_{1}(s)+f(s)^{T} F_{1}(s)\right](u \otimes \psi(f)),\right. \\
& {\left.\left.\left[E_{2}(s)+F_{2}(s) g(s)\right](v \otimes \psi(g))\right)\right\} d s, }
\end{aligned}
$$

where $\left(\right.$, ) denotes the inner product in $h^{d}$.

From (2.5), we obtain the estimate ([8]),

$$
\|M(t)(u \otimes \psi(f))\|^{2} \leqq \int_{0}^{t} \alpha(t, d, f ; r) \Delta(u, f ; r) d r
$$

where

and

$$
\alpha(t, d, f ; r)=(d+1)^{3}\left(1+\|f(r)\|^{2}\right) \exp \left\{t-r+\left(d^{2}+2 d\right) \int_{r}^{t}\|f(s)\|^{2} d s\right\}
$$

$$
\begin{aligned}
& \Delta(u, f ; r)=\max \left\{\left\|E_{j}(r) u \otimes \psi(f)\right\|^{2},\left\|F_{i j}(r) u \otimes \psi(f)\right\|^{2}\right. \\
& \left.\left\|G_{j}(r) u \otimes \psi(f)\right\|^{2},\|H(r) u \otimes \psi(f)\|^{2} ; 1 \leqq i, j \leqq d\right\}
\end{aligned}
$$

\section{Construction of Right Processes}

Let $\left\{M_{i j}, 0 \leqq i, j \leqq d\right\} \subseteq L(\mathscr{D})$ and define

$$
\begin{aligned}
& M_{1}=\sum_{j=1}^{d} M_{0 j} \otimes e_{j}, \\
& M_{2}=\sum_{i, j=1}^{d} M_{i j} \otimes I_{i j}, \\
& M_{3}=\sum_{i=1}^{d} M_{i 0} \otimes e_{j}^{*} \\
& M_{4}=M_{00} .
\end{aligned}
$$


In this section we will establish the conditions under which we can construct extensions $\hat{M}_{i j}$ of each $M_{i j} \otimes I(0 \leqq i, j \leqq d)$ so that there exists a unique solution to the stochastic differential equation.

$$
\begin{aligned}
d V & =d A^{\dagger} \hat{M}_{1} V+d \Lambda \hat{M}_{2} V+\hat{M}_{3} d A V+\hat{M}_{4} V d t, \\
V(0) & =I .
\end{aligned}
$$

The solution $V=(V(t), t \geqq 0)$ to (3.1) (when it exists) will be called a right process to distinguish it from the left processes discussed in Sect. 6 of [13].

We will say that an operator $Y$ with domain $\mathscr{D} \otimes E(S)$ is tensorially finite if there exists $p \in \mathbb{N}$ with

$$
Y=\sum_{k=1}^{p} C_{k} \otimes Y_{k}
$$

where for $1 \leqq k \leqq p$, each $C_{k}\left(Y_{k}\right)$ is an operator on $h_{0}(\Gamma(H))$ with domain $\mathscr{D}(E(S))$.

Lemma 3.1. There exists a sequence $\left(V_{n}, n \in \mathbb{N}\right)$ of stochastic integrals given by the recursion $V_{0}(t)=I$,

$$
V_{n}(t)=I+\int_{0}^{t}\left(d A^{\dagger} M_{1} V_{n-1}+d \Lambda M_{2} V_{n-1}+M_{3} d A V_{n-1}+M_{4} V_{n-1} d t\right)
$$

for each $t \geqq 0$, where each $V_{n}(t)$ is tensorially finite.

Proof. We proceed by induction, noting that each $V_{0}(t)$ is (trivially) tensorially finite. Assume that there exists $p(n) \in \mathbb{N}$ such that for each $t \geqq 0$ there exist adapted square-integrable processes $Y_{k}^{(n)}(t)(1 \leqq k \leqq p(n))$ such that

$$
V_{n}(t)=\sum_{k=1}^{p(n)} C_{k}^{(n)} \otimes Y_{k}^{(n)}(t)
$$

where each $C_{k}^{(n)} \in L(\mathscr{D})$ and is independent of $t$. By (3.5) we obtain

$$
\begin{aligned}
V_{n+1}(t)= & \sum_{k=1}^{p(n)}\left\{\sum_{j=0}^{d} M_{0 j} C_{k}^{(n)} \otimes \int_{0}^{t} Y_{k}^{(n)} d A_{j}^{\dagger}+\sum_{i, j=1}^{n} M_{i j} C_{k}^{(n)} \otimes \int_{0}^{t} Y_{k}^{(n)} d \Lambda_{i j}\right. \\
& \left.+\sum_{j=1}^{n} M_{j 0} C_{k}^{(n)} \otimes \int_{0}^{t} Y_{k}^{(n)} d A_{j}+M_{00} C_{k}^{(n)} \otimes \int_{0}^{t} Y_{k}^{(n)} d s\right\},
\end{aligned}
$$

and since each stochastic integral is again adapted and square-integrable we see that $V_{n+1}(t)$ is tensorially finite and of the same form as $V_{n}(t)$.

Let $\mathscr{C}=\left\{I, M_{i j} ; 0 \leqq i, j \leqq d\right\}$. For any $N \in \mathscr{C}$, we write

$$
N_{i j}=N M_{i j}, \quad 0 \leqq i, j \leqq d .
$$

To avoid notational complexity, it is common practice in quantum stochastic calculus to write the ampliation $X \otimes I$ to the whole of $h$ of an operator $X$ in $h_{0}$ simply as $X$. Although this is harmless when $X$ is bounded, it is misleading in the unbounded case since, for closeable $N, \bar{N} \otimes I \subseteq \overline{N \otimes I}$. We will consequently find it useful to employ the notation

$$
\mathscr{C}^{\otimes}=\left\{X \in L(\mathscr{D} \otimes \Gamma(H), h) ; X=X_{0} \otimes I \text { for some } X_{0} \in \mathscr{C}\right\} .
$$


By Lemma 3.1 we can, for each $N \in \mathscr{C}^{\otimes}$, form the sequence of stochastic integrals $\left(N V_{n}, n \in \mathbb{N}\right)$, where for each $t \geqq 0$,

$$
N V_{n}(t)=N+\int_{0}^{t}\left(d A^{\dagger} N V_{n-1}+d \Lambda N V_{n-1}+N d A V_{n-1}+N V_{n-1} d t\right)
$$

Lemma 3.2. (cf. [13]). For each $N \in \mathscr{C}^{\otimes}, u \in \mathscr{D}, f \in S, n \in \mathbb{N}$ and $t \geqq 0$ we have

$$
\left\|\left(N V_{n}(t)-N V_{n-1}(t)\right)(u \otimes \psi(f))\right\|^{2} \leqq e^{\|f\|^{2}} C(t) \frac{R(t)^{2}}{n !} \Delta_{n}(N, u)^{2},
$$

where

$$
\begin{aligned}
C(t) & =\exp \left\{t+\left(d^{2}+2 d\right) \int_{0}^{t}\|f(u)\|^{2} d u\right\}, \\
R(t) & =(d+1)^{3} t \sup _{0 \leqq s \leqq t}\left(1+\|f(s)\|^{2}\right), \\
\Delta_{n}(N, u) & =\max \cdot\left\{\left\|N_{i_{1}, j_{1}} \cdots N_{i_{n}, j_{n}} u\right\| ; 0 \leqq i_{1}, j_{1}, \ldots, i_{n}, j_{n} \leqq d\right\} .
\end{aligned}
$$

Proof is by induction.

The case $n=1$ follows immediately from (2.6).

To establish the general case, assume (3.7) is valid for some $n$, then (2.6) yields

$$
\begin{aligned}
& \left\|\left(N V_{n+1}(t)-N V_{n}(t)\right)(u \otimes \psi(f))\right\|^{2} \\
& \quad \leqq e^{\|f\|^{2} C(t) \Delta_{n+1}(N, u)^{2}(d+1)^{3}} \int_{0}^{t}\left(1+\|f(s)\|^{2}\right) \frac{R(s)^{n}}{n !} d s,
\end{aligned}
$$

and the result follows from the observation that

$$
\left(1+\|f(s)\|^{2}\right) R(s)^{n} \leqq\left[\sup _{0 \leqq s \leqq t}\left(1+\|f(s)\|^{2}\right)\right]^{n+1} s^{n}
$$

For each $n \in \mathbb{N}, u \in \mathscr{D}$ let $A_{n}(u)=\max \left\{\Delta_{n}(N, u) ; N \in \mathscr{C}\right\}$, and define a linear manifold $\mathscr{D}_{0}$ in $h_{0}$ by

$$
\mathscr{D}_{0}=\left\{u \in \mathscr{D} ; \sum_{n=0}^{\infty} \frac{\rho^{n}}{(n !)^{1 / 2}} A_{n}(u)<\infty \text { for all } \rho>0\right\} .
$$

By (3.4) we have for each $u \in \mathscr{D}_{0}, f \in S, N \in \mathscr{C}^{\otimes}, t \geqq 0$

$$
\sum_{n=1}^{\infty}\left\|\left(N V_{n}(t)-N V_{n-1}(t)\right)(u \otimes \psi(f))\right\| \leqq e^{\|f\|} C(t)^{1 / 2} \sum_{n=1}^{\infty} \frac{R(t)^{n / 2}}{(n !)^{1 / 2}} A_{n}(u)<\infty,
$$

hence each sequence $\left(N V_{n}(t)(u \otimes \psi(f))\right)_{n \in \mathbb{N}}$ converges in $h$ and the convergence is uniform on bounded intervals of $\mathbb{R}^{+}$.

In particular, let $N=I$ and define a process $V=(V(t), t \geqq 0)$ by

$$
V(t)(u \otimes \psi(f))=\lim _{n \rightarrow \infty} V_{n}(t)(u \otimes \psi(f))
$$

for $u \in \mathscr{D}_{0}, f \in S$. It is not difficult to verify that $V$ is adapted and square-integrable.

From now on we will assume that $\mathscr{D}_{0}$ is dense in $h_{0}$ so that each $V(t)$, for $t \geqq 0$ is densely defined. Our next task is to obtain suitable extensions $\hat{N}$ of each 
$N \in \mathscr{C}^{\otimes}$ so that we may establish (3.1). Since each $N \in \mathscr{C}^{\otimes}$ is closeable, we have $V(t)(u \otimes \psi(f)) \in \operatorname{Dom}(\bar{N})$ for each $t \geqq 0, u \in \mathscr{D}_{0}, f \in S$ with

$$
\bar{N} V(t)(u \otimes \psi(f))=\lim _{n \rightarrow \infty} N V_{n}(t)(u \otimes \psi(f)) \text {. }
$$

Define a dense linear manifold $\mathscr{F}$ in $h$ to be the linear span of $(\mathscr{D} \otimes \Gamma(H)) \cup\left\{V(t)(u \otimes \psi(f)) ; t \geqq 0, u \in \mathscr{D}_{0}, f \in S\right\}$, and let $\hat{N}$ be the restriction of $\bar{N}$ to $\mathscr{F}$ so that we have

$$
N \subseteq \hat{N} \subseteq \bar{N}
$$

We are now ready to establish our main result

Theorem 3.3. There exists a unique solution to the stochastic differential equation (3.1),

$$
\begin{aligned}
d V & =d A^{\dagger} \hat{M}_{1} V+d \Lambda \hat{M}_{2} V+\hat{M}_{3} d A V+\hat{M}_{4} V d t, \\
V(0) & =I .
\end{aligned}
$$

Proof. To see that (3.5) defines a solution of (3.1) use (2.5), the Schwarz inequality and the uniformity of convergence in finite intervals of (3.6) to show that for each $u \in \mathscr{D}_{0}, f \in S$,

$$
\begin{aligned}
\lim _{n \rightarrow \infty} & \|\left(\int _ { 0 } ^ { t } \left[d A^{\dagger} \hat{M}_{1}\left(V-V_{n}\right)+d \Lambda \hat{M}_{2}\left(V-V_{n}\right)\right.\right. \\
& \left.+\hat{M}_{3} d A\left(V-V_{n}\right)+\hat{M}_{4}\left(V-V_{n}\right) d t\right)(u \otimes \psi(f)) \|^{2}=0
\end{aligned}
$$

Uniqueness follows by a similar argument to that of [13].

Now let $L_{i j} \in \mathcal{O}(\mathscr{D})(0 \leqq i, j \leqq d)$. We will take

$$
\begin{aligned}
M_{0 j} & =L_{j 0}^{\dagger} \quad(1 \leqq j \leqq d), \\
M_{i j} & =L_{j i}^{\dagger} \quad(1 \leqq i, j \leqq d), \\
M_{j 0} & =L_{0 j}^{\dagger} \quad(1 \leqq j \leqq d), \\
M_{00} & =L_{00}^{\dagger} .
\end{aligned}
$$

For each $u \in \mathscr{D}, n \in \mathbb{N}$, we define

$$
\begin{aligned}
B_{n}(u) & =\max \left\{A_{n}(u), \quad \max \left\{\left\|L_{i_{1}, j_{1}}, \ldots, L_{i_{n}, j_{n}} u\right\| ; 0 \leqq i_{1}, j_{1}, \ldots, i_{n}, j_{n} \leqq d\right\} .\right. \\
\mathscr{D}_{1} & =\left\{u \in \mathscr{D} ; \sum_{n=0}^{\infty} \frac{\rho^{n}}{(n !)^{1 / 2}} B_{n}(u)<\infty \text { for all } \rho>0\right\} .
\end{aligned}
$$

Clearly $\mathscr{D}_{1} \subseteq \mathscr{D}_{0}$. From now on we will assume that $\mathscr{D}_{1}$ is dense in $h_{0}$. Consider the left process given by the stochastic differential equation

$$
\begin{aligned}
d U & =U\left(d A^{\dagger} L_{1}+d \Lambda L_{2}+L_{3} d A+L_{4} d t\right), \\
U(0) & =I .
\end{aligned}
$$

It follows from [13] that there exists a unique solution to (3.8) with each $U(t)$ $(t \geqq 0)$ densely defined on the domain $\mathscr{D}_{1} \otimes E(S)$. 
Corollary 3.4. For each $t \geqq 0, U(t)$ is closeable with

$$
\mathscr{D}_{1} \otimes E(S) \subseteq \operatorname{Dom}\left(U(t)^{*}\right) \text {. }
$$

Furthermore writing $U(t)^{\dagger}=\left.U(t)^{*}\right|_{\mathscr{D}_{1} \otimes E(S)}$ we have

$$
\begin{aligned}
d U^{\dagger} & =d A^{\dagger} \hat{L}_{3}^{\dagger} U^{\dagger}+d \Lambda \hat{L}_{2}^{\dagger} U^{\dagger}+\hat{L}_{1}^{\dagger} d A U^{\dagger}+\hat{L}_{4}^{\dagger} U^{\dagger} d t, \\
U^{\dagger}(0) & =I .
\end{aligned}
$$

Proof. It follows from [13] that for each $t \geqq 0, U(t)$ is the strong limit on $\mathscr{D}_{1}$ of the sequence of iterates defined by

$$
\begin{aligned}
U_{0}(t) & =I, \\
U_{n+1}(t) & =I+\int_{0}^{t} U_{n}\left(d A^{+} L_{1}+d \Lambda L_{2}+L_{3} d A+L_{4} d t\right) .
\end{aligned}
$$

A straightforward inductive argument shows that for each $u, v \in \mathscr{D}_{1}, f, g \in S, n \in \mathbb{N}$

$$
\left\langle V_{n}(t)(u \otimes \psi(f)), v \otimes \psi(g)\right\rangle=\left\langle u \otimes \psi(f), U_{n}(t)(v \otimes \psi(g)\rangle\right.
$$

and the required result follows upon taking limits.

\section{Conditions for Unitarity}

In this section we will, writing $L_{1}=L$, take $L_{2}=W-I, L_{3}=-L^{\dagger} W$ and $L_{4}=i H-\frac{1}{2} L^{\dagger} L$, where $H$ is symmetric in $h_{0}$ and $W$ extends to a unitary operator on $h_{0}^{d}$.

With this choice of coefficients we obtain the following

Lemma 4.1. For each $t \geqq 0, U(t)$ extends to a co-isometry in $h$.

Proof. Using (3.9) in (2.5) we obtain for $t \geqq 0, u, v \in \mathscr{D}_{1}, f, g \in S$

$$
\left.\left\langle U^{\dagger}(t)(u \otimes \psi(f)), U^{\dagger}(t) v \otimes \psi(g)\right)\right\rangle=\langle u \otimes \psi(f), v \otimes \psi(g)
$$

so that, by linearity, $U^{\dagger}(t)$ is isometric on $\mathscr{D}_{1} \otimes E(S)$, and thus extends to an isometry on the whole of $h$.

Since each $\overline{U(t)}$ is co-isometric, $\overline{U^{\dagger}(t)} \overline{U(t)}$ is bounded thus for each $t \geqq 0, f, g \in S$, we may define an operator $T_{f, g}(t) \in B\left(h_{0}\right)$ by the prescription

$$
\left\langle u, T_{f, g}(t) v\right\rangle=\langle\overline{U(t)}(u \otimes \psi(f)), \overline{U(t)}(v \otimes \psi(g))\rangle
$$

for $u, v \in h_{0}$. Now put $u=T_{f, g}(t) v$ in (4.1) and use the Schwarz inequality, the fact that $\left\|U^{\dagger}(t)\right\|=\|U(t)\|=1$ and (2.1) to obtain the estimate

$$
\left\|T_{f, g}(t) v\right\| \leqq e^{\|f\|+\|g\|}\|v\|,
$$

so that

$$
\left\|T_{f, g}(t)\right\| \leqq e^{\|f\|+\|g\|}
$$

for all $t \geqq 0$. Let $Z$ denote the convex set of all maps from $\mathbb{R}^{+}$into $B\left(h_{0}\right)$ which satisfy (4.2). 
Theorem 4.2. For each $t \geqq 0, U(t)$ extends to an isometry in $h$.

Proof. Our strategy will be to prove that for each $t \geqq 0, f, g \in S$,

$$
T_{f, g}(t)=\langle\psi(f), \psi(g)\rangle I .
$$

By (2.5) we have for each $u, v \in \mathscr{D}_{1}$,

$$
\begin{aligned}
& \langle U(t)(u \otimes \psi(f)), U(t)(v \otimes \psi(g))\rangle \\
& =\langle u \otimes \psi(f), v \otimes \psi(g)\rangle+\int_{0}^{t}\left\{\left\langle U(s)(u \otimes \psi(f)), U(s)\left(\overline{f(s)^{T}} L v \otimes \psi(g)\right)\right\rangle\right. \\
& -\left\langle U(s)\left(L^{\dagger} W f(s) u \otimes \psi(f)\right), U(s)(v \otimes \psi(g))\right\rangle \\
& +\left(U(s)\left(f(s)^{T}(W-I) u \otimes \psi(f)\right), U(s)(L v \otimes \psi(g))\right\rangle \\
& +\left\langle U(s)(u \otimes \psi(f)), U(s)\left(\overline{f(s)^{T}}(W-I) g(s) v \otimes \psi(g)\right)\right\rangle \\
& +\left\langle U(s)\left(f(s)^{T}(W-I) \overline{g(s)} u \otimes \psi(f)\right), U(s)(v \otimes \psi(g))\right\rangle \\
& +\left(U(s)\left(f(s)^{T}(W-I) u \otimes \psi(f)\right), U(s)((W-I) g(s) v \otimes \psi(g))\right) \\
& -\left\langle U(s)(u \otimes \psi(f)), U(s)\left(L^{\dagger} W g(s) v \otimes \psi(g)\right)\right\rangle \\
& +\left\langle U(s)\left(g(s)^{T} L u \otimes \psi(f)\right), U(s)(v \otimes \psi(g))\right\rangle \\
& +(U(s)(L u \otimes \psi(f)), U(s)((W-I) g(s) v \otimes \psi(g))) \\
& +\left\langle U(s)\left(\left(i H-\frac{1}{2} L^{\dagger} L\right) u \otimes \psi(f), U(s) v \otimes \psi(g)\right)\right\rangle \\
& +\left\langle U(s)(u \otimes \psi(f)), U(s)\left(\left(i H-\frac{1}{2} L^{\dagger} L\right) v \otimes \psi(g)\right)\right\rangle \\
& +(U(s)(L u \otimes \psi(f)), U(s)(L v \otimes \psi(g)))\} d s .
\end{aligned}
$$

Substituting (4.1) into this expression and simplifying yields

$$
\begin{aligned}
\left\langle u, T_{f, g}(t) v\right\rangle= & \langle u, v\rangle\langle\psi(f), \psi(g)\rangle+\int_{0}^{t}\left\{\left(f(s)^{T} W u, T_{f, g}(s) L v\right)\right. \\
& -\left\langle L^{\dagger} W f(s) u, T_{f, g}(s) v\right\rangle+\left(f(s)^{T} W u, T_{f, g}(s) W g(s) v\right) \\
& -\left(f(s)^{T} u, T_{f, g}(s) g(s) v\right)+\left(L u, T_{f, g}(s) W g(s) v\right) \\
& -\left\langle u, T_{f, g}(s) L^{\dagger} W f(s) v\right\rangle+\left\langle u, T_{f, g}(s)\left(i H-\frac{1}{2} L^{\dagger} L\right) v\right\rangle \\
& \left.+\left\langle\left(i H-\frac{1}{2} L^{\dagger} L\right) v, T_{f, g}(s) v\right\rangle+\left(L u, T_{f, g}(s) L v\right)\right\} d s .
\end{aligned}
$$

Clearly (4.3) is a solution of (4.4). We aim to show that it is the unique solution in $Z$.

Let $T_{f, g}^{\prime}(t)$ be another such solution and define

$$
X_{f, g}(t)=T_{f, g}(t)-T_{f, g}^{\prime}(t) \text { for all } t \geqq 0 .
$$

By making use of estimates of the type

$$
\begin{aligned}
\left|\left(f(s)^{T} W u, X_{f, g}(s) L v\right)\right| & =\left|\sum_{i, j=1}^{d} \overline{f_{i}(s)}\left\langle W_{i j} u, X_{f, g}(s) L_{j} v\right\rangle\right| \\
& \leqq\|f(s)\|\left(\sum_{i=1}^{d}\left(\sum_{j=1}^{d}\left|\left\langle W_{i j} u, X_{f, g}(s) L_{j} v\right\rangle\right|^{2}\right)\right) \\
& \leqq d^{3 / 2}\|f(s)\| \max _{M_{1}, M_{2} \in \mathscr{C}^{\dagger}}\left|\left\langle M_{1} u, X_{f, g}(s) M_{2} v\right\rangle\right|
\end{aligned}
$$

(by the Schwarz inequality), a straightforward inductive argument in (4.4) 
establishes that

$$
\begin{aligned}
\left|\left\langle u, X_{f, g}(t) v\right\rangle\right| \leqq & \frac{C_{f, g}(t)^{n}}{n !} t^{n} \sup _{0 \leqq s \leqq t} \max \left\{\mid\left\langle M_{j_{1}} \cdots M_{j_{n}} u,\right.\right. \\
& \left.\left.X_{f, g}(s) M_{k_{1}} \cdots M_{k_{n}} v\right\rangle \mid ; M_{j_{p}}, M_{k_{q}} \in \mathscr{C}^{\dagger}, 1 \leqq p, q \leqq n\right\}
\end{aligned}
$$

for all $n \geqq 1, t \geqq 0, u, v \in \mathscr{D}_{1}$, where

$$
C_{f, g}(t)=\sup _{0 \leqq s \leqq t}\left\{2 d^{3 / 2}(\|f(s)\|+\|g(s)\|)+\left(d^{2}+1\right)\|f(s)\|\|g(s)\|+d+2\right\} .
$$

Now by the Schwarz inequality and (4.2) we obtain

$$
\begin{aligned}
& \sup _{0 \leqq s \leqq t} \max \left|\left\langle M_{j_{1}} \cdots M_{j_{n}} u, X_{f, g}(s) M_{k_{1}} \cdots M_{k_{n}} v\right\rangle\right| \\
& \quad \leqq \sup _{0 \leqq s \leqq t}\left\|X_{f, g}(s)\right\| \max \left\{\max \left\|M_{j_{1}} \cdots M_{j_{n}} u\right\|^{2}, \max \left\|M_{j_{1}} \cdots M_{j_{n}} v\right\|^{2}\right\} \\
& \quad \leqq\left(\sup _{0 \leqq s \leqq t}\left\|T_{f, g}(s)\right\|+\sup _{0 \leqq s \leqq t}\left\|T_{f, g}^{\prime}(s)\right\|\right) \max \left\{B_{n}(u)^{2}, B_{n}(v)^{2}\right\} \\
& \quad \leqq 2 e^{\|f\|+\|g\|} \max \left\{B_{n}(u)^{2}, B_{n}(v)^{2}\right\} .
\end{aligned}
$$

Combining (4.5) and (4.6) yields

$$
\left|\left\langle u, X_{f, g}(t) v\right\rangle\right| \leqq 2 e^{\|f\|+\|g\|} \frac{C_{f, g}(t)^{n}}{n !} t^{n} \max \left\{B_{n}(u)^{2}, B_{n}(v)^{2}\right\} \rightarrow 0 \quad \text { as } \quad n \rightarrow \infty,
$$

since $u, v \in \mathscr{D}_{1}$.

Hence we deduce that each $X_{f, g}(t)=0$ and our required result follows.

Theorem 4.3. For each $t \geqq 0$, the operator $U(t)$ in the solution of (3.15) has a unitary extension if and only if

$$
L_{2}=W-I, \quad L_{3}=-L_{1}^{\dagger} W, \quad L_{4}=i H-\frac{1}{2} L^{\dagger} L,
$$

where $H$ is a symmetric operator in $h_{0}$ and $W$ extends to a unitary operator on $h_{0}^{d}$.

Proof. Lemma 4.1 and Theorem 4.2 taken together establish sufficiency. Necessity is proved along similar lines to the corresponding result of [13] noting that since our coefficients are unbounded we must use (2.5) in place of the bounded version of the quantum Ito formula.

Note. It is easily verified that the domain $\mathscr{D}_{1}$ is a set of analytic vectors for $L_{4}$, so that if, for example, $H=0$, then by Nelson's theorem, $-\frac{1}{2} L^{\dagger} L$, is essentially self adjoint on $\mathscr{D}_{1}$.

\section{The Construction of Quantum Diffusions}

Let $\mathscr{A}$ be a unital *-subalgebra of $B\left(h_{0}\right) \cap \mathcal{O}(\mathscr{D})$ and $\mathscr{B}$ be a two sided $\mathscr{A}$-module such that

$$
\mathscr{A} \subseteq \mathscr{B} \subseteq \mathcal{O}(\mathscr{D})
$$


An $(\mathscr{A}, \mathscr{B})$-diffusion [4] is a family $\left\{j_{t}, t \geqq 0\right\}$ of injective linear maps from $\mathscr{B}$ into $L(\mathscr{D} \otimes E(S), h)$ satisfying

(i) $\operatorname{Ran}\left(\left.j_{t}\right|_{\mathscr{A}}\right) \subseteq \mathscr{B}(h)$.

(ii) For each $t \geqq 0, x \in \mathscr{A}, y \in \mathscr{B}, j_{t}(y)$ has an extension $j_{t}^{\sim}(y)$ with $\operatorname{Ran}\left(j_{t}(x)\right) \subseteq$ $\operatorname{Dom}\left(j_{t}^{\sim}(y)\right)$ and

$$
\begin{array}{r}
j_{t}^{\sim}(y) j_{t}(x)=j_{t}(y x), \\
j_{t}(x) j_{t}(y)=j_{t}(x y) .
\end{array}
$$

(iii) For each $t \geqq 0, x \in \mathscr{B}$,

$$
j_{t}(x)^{*} \supseteqq j_{t}\left(x^{\dagger}\right) \text {. }
$$

(iv) $j_{0}=\imath \otimes I$, where $\imath$ is the natural embedding of $\mathscr{B}$ into $\mathcal{O}(\mathscr{D})$.

(v) For each $x \in \mathscr{A},\left(j_{t}(x), t \geqq 0\right)$ is adapted and square-integrable.

(vi) There exist linear maps $\alpha: \mathscr{A} \rightarrow \mathscr{A} \otimes \mathbb{C}^{d}, \lambda: \mathscr{A} \rightarrow M_{d}(\mathscr{A}), \tilde{\alpha}: \mathscr{A} \rightarrow \mathscr{A} \otimes\left(\mathbb{C}^{d}\right)^{*}$ and $\tau: \mathscr{A} \rightarrow \mathscr{B}$ such that for each $x \in \mathscr{A}$ and $t \geqq 0$,

$$
d j_{t}(x)=d A^{\dagger} j_{t}(\alpha(x))+d \Lambda j_{t}(\lambda(x))+j_{t}(\tilde{\alpha}(x)) d A+j_{t}(\tau(x)) d t .
$$

$\alpha, \lambda, \tilde{\alpha}$ and $\tau$ are called the structure maps of the diffusion. $\left\{j_{t}, t \geqq 0\right\}$ will be called an $\mathscr{A}$-diffusion when $\mathscr{B}=\mathscr{A}$.

From (i), (ii) and (iii) we observe that each $\left.j_{t}\right|_{\mathscr{A}}$ is a *-homomorphism into $B(h)$. An extensive study of $\mathscr{A}$-diffusions with $\mathscr{D}=h_{0}$ has been carried out by R. L. Hudson and M. E. Evans (see e.g. [12,8,9] and [10]).

Proposition 5.1. (cf. [12]). If $\left\{j_{t}, t \geqq 0\right\}$ is an $(\mathscr{A}, \mathscr{B})$ diffusion then

$$
\alpha(I)=\lambda(I)=\tilde{\alpha}(I)=\tau(I)=0 .
$$

(b) For each $x \in \mathscr{A}$,

(c)

$$
\begin{aligned}
\lambda(x)^{*} & =\lambda\left(x^{*}\right) \\
\tilde{\alpha}(x)^{*} & =\alpha\left(x^{*}\right) \\
\tau(x)^{*} & \supseteqq \tau\left(x^{*}\right) \\
\lambda & =\sigma-l_{d}
\end{aligned}
$$

where $\sigma$ is a unital *-homomorphism from $\mathscr{A}$ into $M_{d}(\mathscr{A})$ and $v_{d}$ identifies $\mathscr{A}$ with the *-subalgebra $\mathscr{A} I$ of $M_{d}(\mathscr{A})$.

$$
\begin{gathered}
\alpha \in \operatorname{Der}_{\sigma}\left(\mathscr{A}, \mathscr{A}^{d}\right), \\
b \tau=\eta,
\end{gathered}
$$

where $b$ is the Hothschild coboundary operator for the complex formed by multilinear maps from $\mathscr{A}$ into $\mathscr{B}$ and the 2-coboundary $\eta$ is given by

$$
\eta(x, y)=-\tilde{\alpha}(x) \alpha(y) \text { for } x, y \in \mathscr{A} .
$$

Proof. To establish $(c)$, the definition of $\left\{j_{t}, t \geqq 0\right\}$ implies that for all $x, y \in \mathscr{A}$, $u, v \in \mathscr{D}, f, g \in S, t \geqq 0$,

$$
\left\langle u \otimes \psi(f), j_{t}(x y)(v \otimes \psi(g))\right\rangle=\left\langle j_{t}(x)^{*}(u \otimes \psi(f)), j_{t}(y)(v \otimes \psi(g))\right\rangle .
$$


Now use (2.5), (5.1), (2.4) and the independence of $d A^{\dagger}, d \Lambda, d A$ and $d t([13,22])$ to obtain (5.6) to (5.8).

(a) and (b) are obtained similarly from $d j_{t}(I)=0$ and (5.2) (respectively)

Equations (5.4) to (5.8) are called the structure equations of the diffusion.

Before we give our construction of $(\mathscr{A}, \mathscr{B})$ diffusions we need to introduce a new concept. Let $\mathscr{E}$ be a linear subspace of $\operatorname{Der}_{\sigma}\left(\mathscr{A}, \mathscr{A}^{d}\right)$. A $\sigma$-connection driven by $\mathscr{E}$ on $\mathscr{D}$ is a linear map

$\nabla: \mathscr{E} \rightarrow \mathscr{L}\left(\mathscr{D}, \mathscr{D}^{d}\right)$ satisfying

$$
\nabla_{\alpha}(a \psi)=\sigma(a) \nabla_{\alpha}(\psi)+\alpha(a) \psi
$$

for all $\alpha \in \mathscr{E}, a \in \mathscr{A}, \psi \in \mathscr{D}$. Notice that if we take $\sigma=l_{d}$ then (5.11) is essentially the notion of connection given in [7]. For a non-trivial example take $x \in \mathscr{A}$, $\sigma(x)=W x W^{\dagger}$, where $W$ is a unitary operator in $M_{d}(\mathscr{A})$ acting on $h_{0}^{d}$, fix $z \in Z(\mathscr{A})^{d}$ and put $\alpha(x)=(\sigma(x)-x) z$ [10].

If we take $\mathscr{E}$ to be the linear span of $\alpha$ then $(W-I) z$ is easily seen to be a $\sigma$-connection.

From now on, we will abuse notation and write $\nabla^{W}=W-I$ even though $\nabla^{W}$ is not strictly a $\sigma$-connection (unless $d=1$ ). A $\sigma$-connection $\nabla$ is said to be semi-balanced if $\nabla_{\alpha}^{j} \in \mathcal{O}(\mathscr{D})$ for each $\alpha \in \mathscr{E}(1 \leqq j \leqq d)$ and balanced if it is semi-balanced and there exists a $*$-homomorphism $\sigma^{\dagger}$ from $\mathscr{A}$ into $M_{d}(\mathscr{A})$, a linear subspace $\mathscr{E}^{\dagger}$ of $\operatorname{Der}_{\sigma}{ }^{\dagger}\left(\mathscr{A}, \mathscr{A}^{d}\right)$ and a $\sigma^{\dagger}$-connection $\tilde{\nabla}$ driven by $\mathscr{E}^{\dagger}$ on $\mathscr{D}$ such that for each $\alpha \in \mathscr{E}$, there exists $\alpha^{\dagger} \in \mathscr{E}^{\dagger}$ for which

$$
\tilde{\nabla}_{\alpha}^{\dagger}=\left(\nabla_{\alpha}^{\dagger}\right)^{T}
$$

It is easily verified that every semi-balanced connection is balanced with $\tilde{\nabla}$ being itself a connection and each

$$
\alpha^{\dagger}=-\tilde{\alpha}^{T}
$$

The $\sigma$-connection $\nabla^{W} z$ discussed above is balanced with $\sigma^{\dagger}(x)=W^{\dagger} x W$ and $\alpha^{\dagger}(x)=\left(\sigma^{\dagger}(x)-x\right) z$ for each $x \in \mathscr{A}$.

Lemma 5.2. Put $d=1$, and let $\sigma$ be invertible, then every semi-balanced $\sigma$-connection is balanced with

$$
\sigma^{\dagger}=\sigma^{-1} \text { and } \alpha^{\dagger}=-\tilde{\alpha}^{\circ} \sigma^{-1} .
$$

Proof. Taking adjoints in (5.17) and restricting to $\mathscr{D}$ yields

$$
\begin{aligned}
a^{*} \nabla_{\alpha}^{\dagger}= & \nabla_{\alpha}^{\dagger} \sigma\left(a^{*}\right)+\alpha(a)^{*} \\
& \nabla_{\alpha}^{\dagger} \sigma\left(a^{*}\right)+\tilde{\alpha}(a)^{*}
\end{aligned}
$$

by (5.5). Now replace a by $-\sigma^{-1}\left(a^{*}\right)$ to get the required result.

We are now ready to construct a class of $(\mathscr{A}, \mathscr{B})$-diffusions.

Let $\mathscr{B}=\mathscr{A} \mathscr{C}$, where the operators in $\mathscr{C}$ are chosen to satisfy the conditions of Theorem 4.3 which guarantee unitarity of $(U(t), t \geqq 0)$. Let $L$ in Sect. 4 be a semi-balanced $\sigma$-connection which we denote $\nabla_{\alpha}$ for some $\alpha \in \operatorname{Der}\left(\mathscr{A}, \mathscr{A}^{d}\right)$ and assume that $H$ is such that $[H, x] \in \mathscr{B}$ for each $x \in \mathscr{A}$. These conditions ensure that $\mathscr{C} \mathscr{A} \subseteq \mathscr{A} \mathscr{C}$ so that $\mathscr{B}$ is indeed a 2 -sided $\mathscr{A}$-module as is required. 
For $a \in \mathscr{A}, N \in \mathscr{C}, t \geqq 0$ we define

$$
j_{t}(a N)=\overline{U(t)} \bar{a} \hat{N} U(t)^{\dagger}
$$

and take $\tilde{j}_{t}(X)=\overline{j_{t}(X)}$ for $X \in \mathscr{B}$. We obtain the following

Theorem 5.3. $\left\{j_{t}, t \geqq 0\right\}$ is an $(\mathscr{A}, \mathscr{B})$-diffusion with structure maps

$$
\begin{aligned}
\sigma(x) & =W x W^{\dagger} \\
\alpha(x) & =\nabla_{\alpha} x-\sigma(x) \nabla_{\alpha} \\
\tau(x) & =i[H, x]-\frac{1}{2} \nabla_{\alpha}^{\dagger} \nabla_{a} x+\nabla_{\alpha}^{\dagger} \sigma(x) \nabla_{\alpha}-\frac{1}{2} x \nabla_{\alpha}^{\dagger} \nabla_{\alpha}
\end{aligned}
$$

for each $x \in \mathscr{A}$.

Proof. The properties of $\nabla_{\alpha}$ and $H$ ensure that $j_{t}$ makes sense on each of the structure maps. Equations (5.16) to (5.18) are obtained by applying (2.5) to

$$
\left\langle U(t)^{\dagger}(u \otimes \psi(f)), x U(t)^{\dagger}(v \otimes \psi(g))\right\rangle
$$

for $u, v \in \mathscr{F}, f, g \in S, t \geqq 0$.

Observe that when $L$ and $H$ extend to bounded operators on $h_{0}$ and $\mathscr{A}=B\left(h_{0}\right)$ then $\left\{\tilde{j}_{t}, t \geqq 0\right\}$ is the diffusion of $B\left(h_{0}\right)$ into $B(h)$ first described in [13].

We note that the unitary process $(U(t), t \geqq 0)$ which induces the diffusion of Theorem 5.3 satisfies

$$
\begin{aligned}
d U & =U\left(d A^{\dagger} \nabla_{\alpha}+d \Lambda \nabla^{W}-\nabla_{\alpha}^{\dagger}\left(\nabla^{W}+I\right) d A+\left(i H-\frac{1}{2} \nabla_{\alpha}^{\dagger} \nabla_{\alpha}\right) d t\right) \\
U(0) & =I .
\end{aligned}
$$

It is not difficult to see that this form is canonical in the sense that any unitary process which induces an $(\mathscr{A}, \mathscr{B})$-diffusion by conjugation must satisfy an equation of the form (5.19). Clearly the diffusion $\left\{j_{t}, t \geqq 0\right\}$ will be an $\mathscr{A}$-diffusion if and only if $\operatorname{Ran}(\tau) \subseteq \mathscr{A}$ (cf. [4]).

The practical application of Theorem 5.2 to construct $(\mathscr{A}, \mathscr{B})$-diffusions is critically dependent upon the existence of semi-balanced $\sigma$-connections. An interesting class of examples where these always exist is obtained by taking $\mathscr{A}$ to be a norm dense *-subalgebra of a $C^{*}$-algebra. If $\omega$ is a faithful state on $\mathscr{A}$, we may realise $\mathscr{A}$ as a ${ }^{*}$-subalgebra of $B\left(h_{0}\right)$, where $h_{0}=L^{2}(\mathscr{A}, \omega)$ and take $\mathscr{D}=\mathscr{A} \psi_{0}$, where $\omega()=.\left\langle\psi_{0}, \cdot \psi_{0}\right\rangle$. Let $\sigma=l_{d}$ and assume that $\alpha \in \operatorname{Der}\left(\mathscr{A}, \mathscr{A}^{d}\right)$ is such that each $\alpha^{j} \in \mathcal{O}(\mathscr{D}), 1 \leqq j \leqq d$. It is easily verified that $\nabla_{\alpha}=\alpha$ defines a balanced connection on $\mathscr{D}$ with $\alpha^{\dagger}$ as given by (5.13). The general set-up is discussed in greater detail in [4] for the case where $d=1$. A specific example will be described in the next section.

By analogy with the case where $\mathscr{D}$ is the space of smooth sections of a complex vector bundle over a compact smooth manifold [2], we call the solution $U=(U(t)$, $t \geqq 0$ ) of (5.19) a quantum stochastic parallel transport process. Equation (5.3) is then called the underlying diffusion of the horizontal lift induced by $U$. 


\section{Examples of Quantum Diffusions}

We will give explicit examples of diffusions which can be constructed using the horizontal lift procedure of Theorem 5.3:

a) Poisson Diffusions ([10,13]). Fix $z \in Z\left(A^{d}\right)$. Then the Poisson process of intensity $z$ is given by

$$
\Pi_{z}=\sum_{i, j=1}^{d} \Pi_{z}^{i j} \otimes I_{i j}^{*}
$$

where

$$
\Pi_{z}^{i j}=\Lambda_{i j}+z_{i} A_{j}^{\dagger}+z_{j}^{*} A_{i}+z_{j}^{*} z_{i} d t
$$

where $z_{i}$ is the $i^{\text {th }}$ component of $z(1 \leqq i \leqq d)$.

Now suppose that $z$ is such that $W z_{j} W^{\dagger}=z_{j}(1 \leqq j \leqq d)$ and take $\alpha(x)=\lambda(x) z$ for $x \in \mathscr{A}$ and $\nabla_{\alpha}=\nabla^{W} z$; then the $\mathscr{A}$-diffusion

$$
d j_{t}(x)=d \Pi_{z} j_{t}(\lambda(x))
$$

underlies the horizontal lift induced by

$$
d U=d \Pi_{z} U \nabla^{W}
$$

Since $\nabla^{W}$ extends to a bounded operator on $h_{0}$, the unitarity of solutions to (6.2) is guaranteed by the theory of [13] and there are no domain conditions to check.

b) Diffusions on the Non-Commutative Two-Torus ([12,15,2,3,4]). Fix $\theta \in \mathbb{R}$ and let $\mathscr{U}_{\theta}$ denote the universal $C^{*}$-algebra generated by two unitaries $S$ and $T$ satisfying

$$
S T=e^{2 \pi i \theta} T S \text {. }
$$

$\mathscr{A}_{\theta}$ will be the norm dense ${ }^{*}$-subalgebra of $\mathscr{U}_{\theta}$ for which $X \in \mathscr{A}_{\theta} \Rightarrow$

$$
X=\sum_{m, n \in \mathbb{Z}} c_{m, n} S^{m} T^{n}
$$

where $\sum_{m, n \in \mathbb{Z}} m^{2 p} n^{2 q}\left|c_{m, n}\right|^{2}<\infty$ for all $m, n \in \mathbb{N}([6,7])$.

The two canonical outer derivatives on $\mathscr{A}_{\theta}$ are $\delta_{1}$ and $\delta_{2}$, where

$$
\left.\begin{array}{ll}
\delta_{1}(S)=2 \pi i S, & \delta_{1}(T)=0 \\
\delta_{2}(S)=0, & \delta_{2}(T)=2 \pi i T
\end{array}\right) .
$$

$\mathscr{A}_{\theta}$ possesses a trace $\omega_{0}$ for which

$$
\omega_{0}\left(X^{*} X\right)=\sum_{m, n \in \mathbb{Z}}\left|c_{m, n}\right|^{2}
$$

for $X$ as in (6.4)

Take $h_{0}=L^{2}\left(\mathscr{A}_{\theta}, \omega_{0}\right)$ and $\mathscr{D}=\mathscr{A} \psi_{0}$ as described at the end of Sect. 5. For $a, b \in \mathbb{C}$, let $\alpha=\alpha \delta_{1}+b \delta_{2}$, fix $d=1$ and put $W=I . \alpha$ and $\alpha^{\dagger}$ as given by (5.13) are mutually adjoint ([4]), hence closeable linear operators on $h_{0}$ with domain $\mathscr{D}$ thus we can take $\nabla_{\alpha}=\alpha$. We aim to show that for each $a, b \in \mathbb{C}$, there is an $(\mathscr{A}, \mathscr{B})$-diffusion 
satisfying, for each $x \in \mathscr{A}$,

$$
d j_{t}(x)=j_{t}(\alpha(x)) d A^{\dagger}+j_{t}(\tilde{\alpha}(x)) d A+j_{t}(\tau(x)) d t
$$

for some $\tau: \mathscr{A} \rightarrow \mathscr{B}$. This will follow immediately from Theorem (5.3) with

$$
\tau(x)=\frac{1}{2} \alpha^{\dagger} \alpha x-\alpha^{\dagger} x \alpha-\frac{1}{2} x \alpha^{\dagger} \alpha
$$

if we can establish the unitarity of solutions to

$$
\begin{aligned}
d U & =U\left(\alpha d A^{\dagger}-\alpha^{\dagger} d A-\frac{1}{2} \alpha^{\dagger} \alpha d t\right), \\
U(0) & =I .
\end{aligned}
$$

Let $\mathscr{A}_{F}$ denote the ${ }^{*}$-subalgebra of $\mathscr{A}_{\theta}$ comprising those operators for which the sum in (6.4) is finite. $\mathscr{A}_{F}$ is also norm-dense in $\mathscr{U}$ [5] thus $\mathscr{A}_{F} \psi_{0} \subset \mathscr{D}$ is dense in $h_{0}$.

The unitarity of solutions to (6.9) will follow immediately if we can show that $\mathscr{A}_{F} \psi_{0}$ is a suitable candidate to be the set $\mathscr{D}_{1}$ of Sect. 3. To see this let $L_{1}=\alpha, L_{2}=\alpha^{\dagger}, L_{3}=-\frac{1}{2} \alpha^{\dagger} \alpha$ and note that, by $(6.5),\left[L_{i}, L_{j}\right]=0(1 \leqq i, j \leqq 3)$ on $\mathscr{D}$. Without loss of generality, take

$$
X=\sum_{m, n=-\ell}^{\ell} c_{m, n} S^{m} T^{n}
$$

and observe that

$$
\alpha(X)=\sum_{m, n=-\ell}^{\ell} 2 \pi i z_{m, n} c_{m, n} S^{m} T^{n},
$$

where $z_{m, n}=a m+b n$. We must show that for all $\rho \geqq 0$,

$$
\sum_{N=0}^{\infty} \frac{\rho^{N}}{\sqrt{N !}} \max \left\{\left\|L L_{j_{1}} \cdots L L_{j_{N}} X \psi_{0}\right\| ; 1 \leqq j_{1}, \ldots, j_{N} \leqq 3\right\}<\infty
$$

for $L \in\left\{I, L_{1}, L_{2}, L_{3}\right\}$. Taking $L=I$, we find that

$$
\begin{aligned}
\max & \left\{\left\|L_{j_{1}} \cdots L_{j_{N}} X \psi_{0}\right\| ; 1 \leqq j_{1}, \ldots, j_{N} \leqq 3\right\} \\
= & \max _{0 \leqq p, q \leqq N}\left\|\alpha^{p}\left(\alpha^{\dagger}\right)^{q}\left(\frac{1}{2} \alpha^{\dagger} \alpha\right)^{N-p-q} X \psi_{0}\right\| \\
= & \max _{0 \leqq p, q \leqq N}\left(\frac{1}{2}\right)^{N-p-q}\left\|\alpha^{N-q}\left(\alpha^{\dagger}\right)^{N-p} X \psi_{0}\right\| \\
= & \left(\frac{1}{2}\right)^{N-p-q}\left\|\sum_{m, n=-\ell}^{\ell}(2 \pi i)^{2 N-p-q} z_{m, n}{ }^{N-q} \overline{z_{m, n}}{ }^{N-p} C_{m, n} S^{m} T^{n}\right\| \\
& \leqq\left(2 \pi^{2}\right)^{N} M_{N, l}(a, b),
\end{aligned}
$$

where

$$
M_{N, \ell}(a, b)=\max _{0 \leqq p, q \leqq N}\left(\sum_{m, n=-\ell}^{\ell}\left|z_{m, n}\right|^{4 N-2 p-2 q}\left|C_{m, n}\right|^{2}\right)^{1 / 2}
$$

and we have used (6.10), (6.6) and the fact that

$$
\left\|Y \psi_{0}\right\|^{2}=\omega_{0}\left(Y^{*} Y\right) \text { for } Y \in \mathscr{A}_{F} .
$$


Now there exist subsets $P_{\ell}$ and $Q_{\ell}$ of $\{n \in Z,|n| \leqq \ell\}^{2}$ such that $\left|z_{m, n}\right|<1$ for all $(m, n) \in Q_{\ell}$ and $\left|z_{m, n}\right| \geqq 1$ for all $(m, n) \in P_{\ell}$. (Of course, it may be that one of these subsets is empty.) Hence, by (6.6),

$$
\begin{aligned}
M_{N, \ell}(a, b)= & \max _{0 \leqq p, q \leqq N}\left(\sum_{(m, n) \in P_{\ell}}\left|z_{m, n}\right|^{4 N-2 p-2 q}\left|C_{m, n}\right|^{2}\right. \\
& \left.+\sum_{(m, n) \in Q_{\ell}}\left|z_{m, n}\right|^{4 N-2 p-2 q}\left|C_{m, n}\right|^{2}\right)^{1 / 2} \\
\leqq & \left(\sum_{(m, n) \in P_{\ell}}\left|z_{m, n}\right|{ }^{4 N}\left|C_{m, n}\right|^{2}+\sum_{(m, n) \in Q_{\ell}}\left|C_{m, n}\right|^{2}\right)^{1 / 2} \\
\leqq & \left(\sum_{m, n=-\ell}^{\ell}\left|z_{m, n}\right|^{4 N}\left|C_{m, n}\right|^{2}+\left\|X \psi_{0}\right\|^{2}\right)^{1 / 2} \\
\leqq & {\left[(\ell|a+b|)^{4 N}+1\right]^{1 / 2}\left\|X \psi_{0}\right\| . }
\end{aligned}
$$

Now

$$
\begin{aligned}
\sum_{N=0}^{\infty} \frac{\rho^{N}}{\sqrt{N !}} M_{N, \ell}(a, b) & \leqq \sum_{N=0}^{\infty} \frac{\rho^{N}}{\sqrt{N !}}\left[1+(\ell|a+b|)^{4 N}\right]^{1 / 2}\left\|X \psi_{0}\right\|^{2} \\
& \leqq \sum_{N=0}^{\infty} \rho^{N}\left(\frac{2}{N !} \max \left(1,(\ell|a+b|)^{4 N}\right)\right)^{1 / 2}\left\|X \psi_{0}\right\|^{2}
\end{aligned}
$$

$<\infty$ as required. The general result $(6.11)$ is established similarly.

An analysis of the conditions under which (6.9) yields an $\mathscr{A}_{\theta}$ diffusion driven by connections minimising the Yang-Mills functional of [7] were studied in [2] and [3]. A rigorous proof of the unitarity of the parallel transport processes for these diffusions was given in [8] using a different, (less general) technique to that described here (see also [21]).

Acknowledgement. I would like to thank Robin Hudson for pointing out several errors in an earlier version of part of this paper and the referee for many helpful suggestions.

Note. Since this paper was completed, I have received the preprint [20] in which a similar result to Theorem 4.3 below is established using different techniques, for the case of one-dimensional noise. More recently [21] has appeared which gives another proof of this theorem, again for one-dimensional noise, with an improved estimate.

\section{References}

1. Accardi, L., Schurmann, M., von Waldenfels, W.: Quantum Independent Increment Processes on Superalgebras. Math. Z. 198, 451-77 (1988)

2. Applebaum, D.: Quantum stochastic parallel transport processes on non-commutative vector bundles. Lecture Notes in Mathematics, vol. 1303, pp. 20-37. Berlin, Heidelberg, New York: Springer 1988

3. Applebaum, D.: Stochastic evolution of Yang-Mills connections on the non-commutative two-torus, Lett. Math. Phys. 16, 93-99 (1988)

4. Applebaum, D.: Quantum diffusions on involutive algebras. Lecture Notes in Mathematics, vol. 1442, pp. 70-85. Berlin, Heidelberg, New York: Springer 1990 
5. Bratteli, O.: Derivations, dissipations and group actions. Lecture Notes in Mathematics vol. 1229. Berlin, Heidelberg, New York: Springer 1986

6. Connes, A.: Non-commutative differential geometry. IHES Publ. Math. 62, 41-144 (1985)

7 Connes, A., Rieffel, M.: Yang-Mills for non-commutative two-tori. AMS Contemporary Mathematics 62, 237-67 (1987)

8. Evans, M. E.: Quantum diffusions, Nottingham University PhD thesis (1988)

9. Evans, M. E.: Existence of quantum diffusions. Probab. Th. Rel. Fields 81, 473-83 (1989)

10. Evans, M. E., Hudson, R. L.: Multidimensional quantum diffusions. Lecture Notes in Mathematics, vol. 1303, pp. 69-89. Berlin, Heidelberg, New York: Springer 1988

11. Holevo, A. S.: Time-ordered exponentials in quantum stochastic calculus. Steklov Math. Inst. preprint (1988)

12. Hudson, R. L.: Algebraic theory of quantum diffusions. Lecture Notes in Mathematics vol. 1325, pp. 113-25. Berlin, Heidelberg, New York: Springer 1988

13. Hudson, R. L., Parthasarathy, K. R.: Quantum Ito's formula and stochastic evolution. Commun. Math. Phys. 93, 301-23 (1984)

14. Hudson, R. L., Parthasarathy, K. R.: Stochastic dilations of uniformly continuous, completely positive semigroups. Acta Appl. Math. 2, 353-78 (1984)

15. Hudson, R. L., Robinson, P.: Quantum diffusions and the non-commutative torus. Lett. Math. Phys. 15, 47-53 (1988)

16. Ikeda, N., Watanabe, S.: Stochastic differential equations and diffusion processes. North-Holland Kodansha 1981

17. Lassner, G.: Topological algebras of operators. Rep. Math. Phys. 3, 279-93 (1972)

18. Parthasarathy, K. R.: Quantum stochastic calculus. Lecture Notes in Mathematics vol. 1203, pp. 177-96. Berlin, Heidelberg, New York: Springer 1986

19. Robinson, P.: Quantum diffusions on the rotation algebras and the quantum Hall effect. Lecture Notes in Mathematics, vol. 1442, pp. 326-33, Berlin, Heidelberg, New York: Springer 1990

20. Vincent-Smith, G. F.: Unitary quantum stochastic evolutions. Proc. London Math. Soc. 63, 1-25 (1991)

21. Fagnola, F.: On quantum stochastic differential equations with unbounded coefficients. Prob. Th. Rel. Fields. 86, 501-17 (1990)

22. Lindsay, J. M.: Independence for quantum stochastic integrators. Nottingham University preprint (1989)

Communicated by A. Connes 